\title{
Triply Differential Single Ionization of Argon: Charge Effects for Positron and Electron Impact
}

\author{
O. G. de Lucio \\ Instituto de Física, Universidad Nacional Autónoma de México, Apartado Postal 20-364 01000, México DF, Mexico \\ S. Otranto \\ CONICET and Departamento de Física, Universidad Nacional del Sur, 8000 Bahía Blanca, Argentina \\ R. E. Olson and R. D. DuBois \\ Department of Physics, Missouri University of Science and Technology, Rolla, Missouri 65409, USA
}

(Received 27 August 2009; published 23 April 2010)

\begin{abstract}
Triply differential single ionization of Ar by $200 \mathrm{eV}$ positron and electron impact is measured and calculated. For an unequivocal test of kinematic differences, fully differential ejected electron angular distributions are measured using the same experimental apparatus and conditions for both positron and electron impact. The binary/recoil intensity ratios are shown to significantly differ for the two projectiles. These data are used to test theoretical calculations.
\end{abstract}

DOI: 10.1103/PhysRevLett.104.163201

PACS numbers: 34.80.Dp, 34.10.+x

Introduction.-Ever since the discovery of the positron, questions about how antimatter-matter interactions are similar to, or different from, matter-matter interactions have been asked. The majority of studies performed to address these questions have compared elastic and inelastic processes resulting from positron and electron impact on gaseous or condensed phase targets [1]. For dilute gases individual interactions can be studied, but until recently weak beam intensities limited positron based experiments to studies of total cross sections. These have shown that the total single ionization cross sections for positron and electron impact agree within $10 \%$ at energies above $200 \mathrm{eV}$ [2]. Similar behavior is also observed for single ionization by high energy protons and antiprotons [3]. At lower energies, total cross section studies have shown that changing the sign of the projectile charge influences the cross section because of electron capture or positronium formation processes, target polarization, and trajectory effects.

First-order perturbation theories used to describe these interactions predict identical total and differential cross sections for fast matter and antimatter impact, whereas more sophisticated treatments show distinct differences in the differential electron emission measured as a function of the momentum transfer. Early examples of these predicted differences were triply differential ionization cross section (TDCS) calculations for ionization of helium and atomic hydrogen [4-6]. Theory predicted that the binary electron emission (interactions where the other bound electrons and target nucleus act as spectators) is enhanced (decreased) for positron (electron) impact while the recoil intensity (interactions where the ejected electron also interacts with the target nucleus as it leaves) demonstrates opposite effects. Theory also predicted that, depending on the sign of the charge, the directions of the binary and recoil lobes would shift with respect to the momentum transfer direction. Recent calculations for ionization of $\mathrm{H}_{2}$ predict similar features [7].

However, prior to the present study these predictions remained untested. Comparisons of experiment and theory were limited to electron impact, i.e., tests of matter-matter interactions. For antimatter-matter interactions, only zero degree electron emission data were available [8] and such data do not provide information about the binary and recoil interactions which are the dominant components of the cross section. In this Letter, the first direct comparison of positron and electron impact fully kinematic data measured for a range of electron emission angles and energies is presented. By using identical experimental conditions for both positron and electron impact, uncertainties associated with different apparatuses and techniques are minimized. Thus, after decades, long-standing theoretical predictions can finally be tested.

This study provides the most stringent test to date of theoretical predictions of similarities and differences in inelastic antimatter-matter and matter-matter interactions and dynamics. With regard to atomic interactions, the present work compares how momentum transfer data are influenced by electron exchange (or lack of), polarization effects, and postcollision interactions by comparing positron and electron impact data. More broadly, conclusions derived from the present study impact our understanding of positron interactions, which is important in fields where positrons play important roles, e.g., in the production of antihydrogen and other antiparticles and the subsequent fundamental studies of QED, CPT, gravitational forces on antimatter [9], tomographic imaging in medicine [10,11], characterization of materials, and ionization of biological molecules. With regard to medical and biological effects, knowledge about positron interactions is recognized as being important in improving the spatial resolution of 
positron-emission tomography images [12] plus in accounting for differences in the axial and radial penetrations and doses measured for positron and electron impact [13]. Additional examples of the role positrons play in many fields can be found in Ref. [14].

Two types of triply differential data are presented for $200 \mathrm{eV}$ positron and electron impact single ionization of argon. One presents TDCS data in the traditional form of ejected electron angular distributions measured as a function of momentum transfer. This provides information about and tests predictions of differences in the relative intensities and directions of electrons emitted in binary and recoil interactions. The other presents TDCS data as a function of projectile scattering angle and energy loss and for electrons emitted into a broad range of angles. This provides information about the relative intensities for binary and recoil interactions as functions of energy loss and scattering angle.

Experimental method and data analysis.-The experimental device and techniques employed for this work are described elsewhere $[15,16]$. The important features for the present study are that both the positron and the electron beams are injected along the same path and are collimated by the same aperture as they enter the interaction chamber. Target ions produced in the beam-gas jet overlap are extracted by a $1.2 \mathrm{~V} / \mathrm{cm}$ electric field and detected by a channel electron multiplier. Opposite the recoil detector is a position sensitive channel plate detector (PSD) sensitive to ejected electrons with geometric emission angles, $\theta_{e}$, $\varphi_{e}$, between $30^{\circ}$ and $150^{\circ}$ measured along the beam direction. The influence of the extraction field on the angles and acceptance ranges for low ejected electron energies plus changes in the solid angle and transmission of the grids in front of the detector as functions of the emission angle (see Refs. [15,16] for details) were modeled and used to convolute theory over experimental parameters for comparison purposes. Forward scattered projectiles are energy analyzed by means of an electrostatic analyzer and are detected by a second PSD. An aperture limits the horizontal scattering angles, $\varphi_{p}$, to $0^{\circ} \pm 2.4^{\circ}$ while the projectile PSD limits the vertical scattering angles, $\theta_{p}$, to $0^{\circ} \pm 7.5^{\circ}$.

Time-of-flight and two-dimensional position data for the scattered projectile and the ejected electron were measured using scattered projectile-ejected electron-recoil ion coincidences and recorded in list-mode fashion. Fully kinematic, e.g., TDCS, information was obtained for the ejected electron by imposing conditions on the time-offlight spectra, to ensure single ionization had occurred, and on the projectile energy loss and scattering angles. A convolution of the beam profile across the energy loss window provided information about the range of energy losses which contribute.

These TDCS data were binned every $5^{\circ}$ along the beam direction and $\pm 20^{\circ}$ perpendicular to the beam to improve statistics. Finally, the positron impact data were normal- ized to the electron impact data using the total scattered projectile intensities recorded for scattering angles and energy losses less than approximately $\pm 7^{\circ}$ and $35 \mathrm{eV}$, respectively. This includes the majority of ionization events, and according to Ref. [2], the single ionization cross sections at $200 \mathrm{eV}$ are within $10 \%$ of each other, which is smaller than most of our statistical uncertainties.

Calculations, using the Continuum Distorted WaveEikonal Initial State (CDW-EIS) method [17] in its postform, have also been performed for the same experimental parameters as those measured. This model which reduces a multielectronic target to an effective three body problem in which only one bound electron is considered has recently been used to study electron-argon collisions [18]. Briefly, the method considers the initial state correlation by employing eikonal factors to represent the interactions between the projectile and the atomic core and bound electron. The ejected electron interaction with the target ion is modeled by a static screening term plus a local approximation for the exchange term [19].

Results and discussion.-Figures 1(a) and 1(b) present an example of our measured fully differential data for $200 \mathrm{eV}$ positron [filled symbols in Fig. 1(a)] and electron [open symbols in Fig. 1(b)] impact for a scattering angle of $3^{\circ} \pm 1^{\circ}$ and an energy loss of $21.2 \pm 3 \mathrm{eV}$, corresponding to ejected electron energies of $5.4 \pm 3 \mathrm{eV}$. Normalized counts with statistical uncertainties are plotted versus the geometric electron emission angle which for this ejected electron energy is approximately the same as the emission angle. The solid curves are third-order polynomial fits to the experimental data. The dashed curves are CDW-EIS

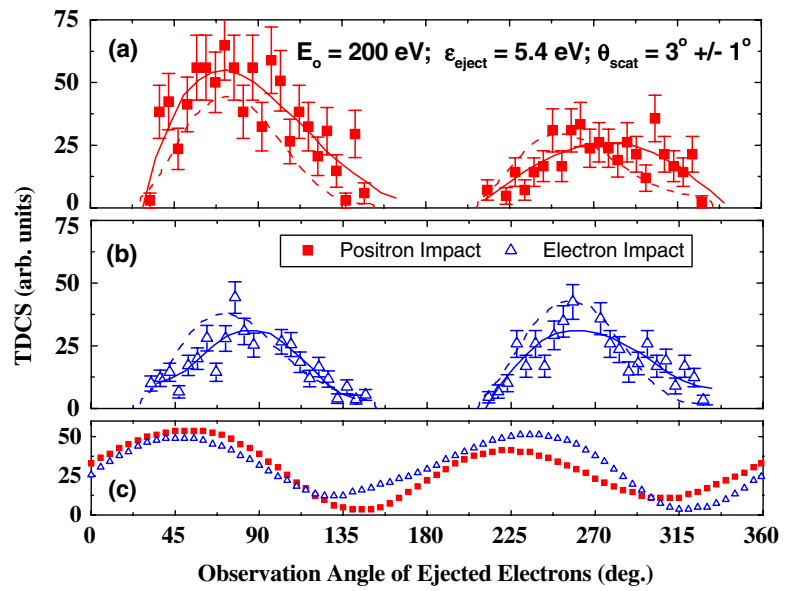

FIG. 1 (color online). Triply differential $5.4 \mathrm{eV}$ electron emission distributions for single ionization of argon by $200 \mathrm{eV}$ positrons [experiment (a), CDW-EIS theory (c)] and electrons [experiment (b), CDW-EIS theory (c)] which have been scattered by $3^{\circ}$. Red filled squares are for positron impact, and blue open triangles are for electron impact; the solid curves are polynomial fits to the data, and the dashed curves are the CDW-EIS theory from (c) convoluted over experimental parameters. The unconvoluted and convoluted theory curves have each been normalized to the electron impact experimental fit at $90^{\circ}$. 
calculations convoluted over experimental parameters. Figure 1(c) shows our unconvoluted theoretical TDCS predictions for $5 \mathrm{eV}$ electron emission and a projectile scattering angle of $3^{\circ}$. The filled and open symbols are for positron and electron impact, respectively. A single normalization between experiment and theory has been used; namely, the convoluted theoretical cross sections are normalized at $90^{\circ}$ to the curve fitted to the experimental electron impact data. This fixes the binary and recoil structure and relative magnitudes between experiment and theory in Figs. 1(a) and 1(b). The reader is reminded that the experimental data are also fixed relative to one another by a single normalization.

In performing the convolution of the theoretical cross sections over experimental parameters, we found a fairly broad Gaussian profile of energy losses contribute to our experimental TDCS and we do not have theoretical values for all energy losses. However, tests showed that because of the reduced contributions away from the Gaussian centroid, convolutions performed using only the centroid energy TDCS or different combinations of available and interpolated TDCS at other energies all yielded essentially the same results. Hence, the (dashed) curves in Figs. 1(a) and 1(b) are a convolution of the $5 \mathrm{eV}$ theory in Fig. 1(c) over our experimental parameters. We see that the convolution severely alters the original distributions by truncating angles in the extreme forward (approaching $0^{\circ}$ and $360^{\circ}$ ) and backward (approaching $180^{\circ}$ ) directions. The truncation primarily arises because of solid angle effects, e.g., projecting emission solid angles onto a planar detector.

A comparison of the experimental positron and electron impact data shows that binary events (data for angles less than $180^{\circ}$ ) are significantly larger for positron impact, in accordance with theoretical predictions [ [16] and Fig. 1(c)]. The same is found for other energy losses and scattering angles which are not shown here. For recoil events, the relative intensity for positron and electron impact is observed to depend on the energy loss and scattering angle; e.g., when the energy losses are relatively small (large) and the scattering angles are large (small), the recoil intensity is somewhat larger (smaller) for positron impact. Between these extremes the recoil intensities are roughly equivalent for positron and electron impact. The fitted curves in Figs. 1(a) and 1(b), plus data for other energy losses and scattering angles, indicate that the binary and recoil lobes for positron impact are slightly more in the forward and backward directions (closer to the direction of the beam) than they are for electron impact.

A comparison of experiment and the convoluted theoretical results shows that our CDW-EIS theory does an excellent job in predicting the approximately equal binary and recoil peak intensities that we observe for electron impact. Our theoretical model also predicts the qualitative enhancement observed for the positron impact binary intensity as well as its slightly reduced recoil intensity. This behavior is in accordance with previous Born-3C calcula- tions in $e^{+}$and $e^{-}$impact ionization of $\mathrm{H} \mathrm{[6]} \mathrm{where} \mathrm{it} \mathrm{has}$ been shown that the height of the binary peak is mostly affected by the projectile-electron interaction in the final channel. However, it underestimates the relative magnitude of the enhancement for binary interactions plus does not reproduce the relative shift that we observe. Our model predicts that the recoil intensity is more backward directed than is observed. This demonstrates that a CDW-EIS model is capable of predicting the general qualitative features but that improvements are still required.

TDCS information was also obtained for the projectile scattering channel. This was done by measuring the projectile scattering angle and energy loss in coincidence with singly charged argon ions and electrons emitted for the full range of $\varphi_{e}$ angles, i.e., by measuring $d^{3} \sigma\left(\Delta E, \Delta \theta_{p}, 30^{\circ}<\varphi_{e}<150^{\circ}\right)$. To remove any possible scattering asymmetries, these yields were divided by doubly differential yields for projectile scattering and energy loss, i.e., $d^{2} \sigma\left(\Delta E, \Delta \theta_{p}\right)$, which were measured simultaneously. The ratio, shown in Fig. 2, provides information about the relative contributions of binary and recoil interactions as functions of energy loss and scattering angle.

The upper portion of Fig. 2 shows these results as a function of scattering angle for an energy loss of $21 \pm$ $3 \mathrm{eV}$, which is the same as for the data shown in Fig. 1. Positive and negative scattering angles indicate recoil and binary events, respectively. The red solid symbols and fitted solid curve are for positron impact; the blue open symbols and blue curve are for electron impact. The data shown here plus other data for larger energy losses illus-
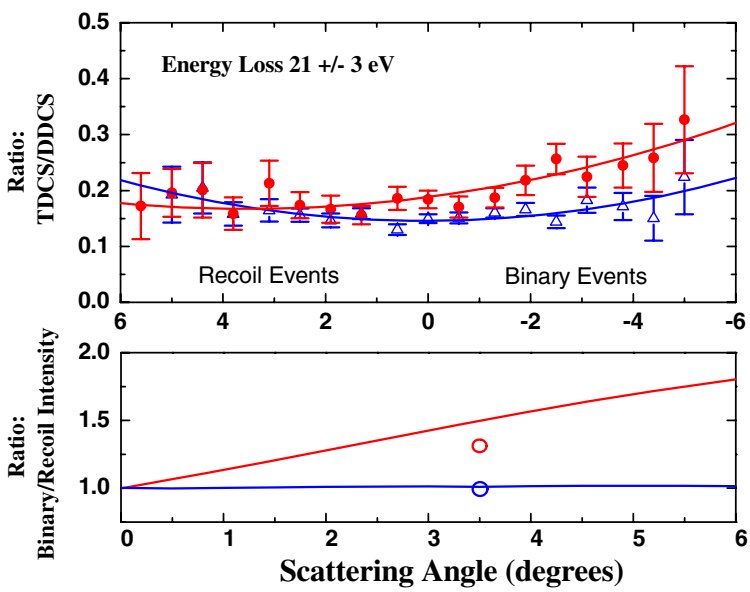

FIG. 2 (color online). Upper portion: Ratio of triply to doubly differential yields measured for projectile scattering. Data are for single ionization of $\mathrm{Ar}$ by $200 \mathrm{eV}$ positrons (filled red circles) and electrons (open blue triangles) and an energy loss corresponding to the ejected electron energies shown in Fig. 1. The solid red and blue lines are fits to the experimental data. Positive angles indicate recoil interactions; negative angles indicate binary interactions. Lower portion: Ratio of binary to recoil interactions from the data shown in the upper portion. Solid lines are from the fits to the experimental data; the open symbols are our CDW-EIS calculations. 
trate two features. One is that binary events become increasingly more important with increasing scattering angle. The other, best illustrated by the positron data in Fig. 2, is that the importance of recoil events for both projectiles remains relatively constant as a function of scattering angle. This means that the probability of binary to recoil events systematically increases with scattering angle as shown in the lower portion of Fig. 2 where we have divided the values of the fitted curve at positive angles by the values at corresponding negative angles. The open symbols in the lower portion are ratios obtained from our CDW-EIS calculations. In this case since binary to recoil intensity ratios are used, no normalization between experiment and theory is required. Again, qualitative but not quantitative agreement is seen for a $3.5^{\circ}$ scattering angle.

The relative enhancement for positron with respect to electron impact for binary interactions could result from (a) impact parameter changes resulting from precollision interactions between the incoming projectile and the partially screened target nucleus, (b) reduced binding of the atomic electrons because of the attractive potential of the incoming positrons, and/or (c) different interaction probabilities due to polarization of the target electron cloud by the incoming projectile. For recoil interactions, a recent theoretical study [18] of electron impact ionization of argon indicated that strong interactions and momentum exchange between the ion core and the emitted electron play a significant role in the recoil structure and that theory has difficulty in modeling this. In our data, the momentum transfer is the same for positron and electron impact, which might explain why we observe similar recoil intensities in contrast to theoretical predictions.

In conclusion, long-standing theoretical predictions of fully differential single ionization data resulting from positron and electron impact have been tested. The predicted relative enhancement for positron impact binary interactions has been confirmed, both in the electron emission and the projectile scattering channel. However, for recoil interactions roughly equal intensities for positron and electron impact are observed, whereas theory predicts an enhancement for electron impact. The experimental data also indicate that the binary and recoil emissions are shifted to more forward and backward directions, respectively, for positron impact, which may indicate that postcollision effects between the scattered projectile and ejected electron are important at this impact energy. In general, comparisons of the present data with CDW-EIS calculations show that for electron impact ionization theory does a good job in describing the binary and recoil intensities and a reasonable job in describing the angular behavior. However, the present theory tends to underestimate the relative enhancement of the binary peak for positron impact plus does not predict the relative shift of the binary peak direction that is observed. More importantly, it pre- dicts features at larger momentum transfers that are inconsistent with extrapolations from the data presented here. It is possible that the nonspherically symmetric structure of the argon $3 p_{0}$ and $3 p_{1}$ subshells may be responsible for some of these differences (see Ref. [18]). Another possibility is that multiple ionization might influence the "effective" lower impact parameter for the single ionization channel. Additional data and comparisons of the type presented here are needed to answer these questions.

As a final note, recent advancements in antiproton experiments [20] indicate that within the next decade similar comparisons between proton and antiproton impact can be made. Those data, plus positron and electron impact data such as in the present study, will allow very detailed investigations to be made of both charge and mass effects on the interaction dynamics.

The experimental work was supported by the National Science Foundation and by UNAM-PAPIIT-IN105510; the theoretical work was supported by PGI 24/F038, PICT2007-00887 of the ANPCyT, and PIP 112-200801-02760 of CONICET (Argentina).

[1] G. Laricchia et al., J. Phys. Conf. Ser. 194, 012036 (2009).

[2] H. Knudsen et al., J. Phys. B 23, 3955 (1990).

[3] K. Paludan et al., J. Phys. B 30, 3951 (1997).

[4] S. Sharma and M. K. Srivastava, Phys. Rev. A 38, 1083 (1988).

[5] M. Brauner and J. S. Briggs, J. Phys. B 26, 2451 (1993).

[6] J. Berakdar, J. S. Briggs, and H. Klar, J. Phys. B 26, 285 (1993).

[7] Á. Benedek and R. I. Campeanu, Nucl. Instrum. Methods Phys. Res., Sect. B 266, 458 (2008).

[8] Á. Kövér and G. Laricchia, Phys. Rev. Lett. 80, 5309 (1998).

[9] G. Gabrielse, Adv. At. Mol. Opt. Phys. 50, 155 (2005).

[10] B. Feng et al., IEEE Trans. Med. Imaging 25, 838 (2006).

[11] B. J. Fueger et al., Molecular Imaging and Biology 11, 269 (2009).

[12] C. Champion and C. Le Loirec, Phys. Med. Biol. 51, 1707 (2006).

[13] S. M. Pimblott and L.D. A. Siebbeles, Nucl. Instrum. Methods Phys. Res., Sect. B 194, 237 (2002).

[14] G. Laricchia et al., Radiat. Phys. Chem. 68, 21 (2003).

[15] O. G. de Lucio, J. Gavin, and R. D. DuBois, Phys. Rev. Lett. 97, 243201 (2006).

[16] R. D. DuBois, O. G. de Lucio, and A.C.F. Santos, in Radiation Physics Research Progress, edited by A. N. Camilleri (Nova, New York, 2008).

[17] P. D. Fainstein, V. H. Ponce, and R. D. Rivarola, J. Phys. B 24, 3091 (1991).

[18] S. Otranto, Phys. Rev. A 79, 012705 (2009).

[19] F. A. Gianturco and S. Scialla, J. Phys. B 20, 3171 (1987).

[20] H. Knudsen, J. Phys. Conf. Ser. 194, 012040 (2009). 